\title{
Shrinking Droplets in Electrospray Ionization and Their Influence on Chemical Equilibria
}

\author{
Arno Wortmann, Anna Kistler-Momotova, and Renato Zenobi \\ Department of Chemistry and Applied Biosciences, ETH Zurich, Zurich, Switzerland \\ Martin C. Heine, Oliver Wilhelm, and Sotiris E. Pratsinis \\ Department of Mechanical and Process Engineering, ETH Zurich, Zurich, Switzerland
}

We investigated how chemical equilibria are affected by the electrospray process, using simultaneous in situ measurements by laser-induced fluorescence (LIF) and phase Doppler anemometry (PDA). The motivation for this study was the increasing number of publications in which electrospray ionization mass spectrometry is used for binding constant determination. The PDA was used to monitor droplet size and velocity, whereas LIF was used to monitor fluorescent analytes within the electrospray droplets. Using acetonitrile as solvent, we found an average initial droplet diameter of $10 \mu \mathrm{m}$ in the electrospray. The PDA allowed us to follow the evolution of these droplets down to a size of $1 \mu \mathrm{m}$. Rhodamine B-sulfonylchloride was used as a fluorescent analyte within the electrospray. By spatially resolved LIF it was possible to probe the dimerization equilibrium of this dye. Measurements at different spray positions showed no influence of the decreasing droplet size on the monomer-dimer equilibrium. However, with the fluorescent dye pair DCM and oxazine 1 it was shown that a concentration increase does occur within electrosprayed droplets, using fluorescence resonance energy transfer as a probe for the average pair distance. (J Am Soc Mass Spectrom 2007, 18, 385-393) @ 2007 American Society for Mass Spectrometry

$\mathrm{F}$ or more than 10 years now, electrospray ionization (ESI) has been used for the analysis of noncovalent complexes [1]. Although these studies have been extended to quantitative measurements of binding affinities of noncovalent complexes [2-4], it is still not clear to what extent the relative yield of gas-phase ions produced by ESI represents their relative solution concentrations. If electrospray ionization mass spectrometry (ESI-MS) is to be used to determine binding constants of noncovalent complexes, this question is of profound significance.

An important aspect of the highly dynamic electrospray process concerns the shrinking droplets. After the droplet creation, solvent evaporation and Coulomb explosions lead to a decrease of droplet size until all solvent has evaporated and the final desolvated and ionized analytes are produced. A decrease of droplet size resulting from solvent evaporation will lead to a concentration increase of nonvolatile analytes within the droplets. For dissolved species that participate in a chemical equilibrium, such as free and bound states of partners that can form a noncovalent complex, the question arises whether the increasing concentrations affect the equilibrium position. Given that binding

Published online November 16, 2006

Address reprint requests to Prof. Renato Zenobi at ETH Zurich, Department of Chemistry and Applied Biosciences, ETH Honggerberg, HCI E 329, CH-8093 Zurich, Switzerland. E-mail: zenobi@org.chem.ethz.ch constant determination with ESI-MS usually relies on measuring the relative amounts of free and bound species, a perturbation of the equilibrium introduced by the shrinking droplets would influence the results of such an experiment. Recent studies show that the time between droplet creation and production of desolvated ions in ESI is within milliseconds [5]. In other words, if the association and dissociation reactions are fast, an equilibrium should be able to track a concentration increase within the droplets.

The shrinking droplet volume also leads to Coulomb explosions/droplet fission, when the mutual repulsion of charges is high enough to overcome the surface tension of the droplet. The parent droplet ejects several smaller offspring droplets, which together carry $10-25 \%$ of the charge, but only about $5 \%$ of the mass of the original droplet [9]. These offspring droplets then segregate within the electrospray, as shown theoretically by Wilhelm et al. [10]. Analytes will not generally be evenly distributed between the parent and offspring droplets. Partitioning processes, which have been investigated by several research groups [6-8], create a different analyte concentration in parent and offspring droplets. Charged and surface active analytes, which are preferentially residing at the droplet surface, have the tendency to accumulate in the offspring droplets [11]. Although diffusion can act as a counteractive process, some compounds will be enriched in the offspring droplets, whereas others will be depleted. Effects 
arising from asymmetric partitioning would be averaged out if analytes from all droplets were ionized and sampled by the mass spectrometer. However, ion production and sampling from smaller droplets is expected to be much more effective. The ion production pathway should therefore exert an influence on the relative amounts of free and complexed species that are ultimately detected by the mass spectrometer. Different models have been proposed for ESI ion formation: the ion evaporation model (IEM) [12] suggests production of ions at an early stage in the ESI plume, that is, the time for an equilibrium to react to changes in droplet volume is short, within microseconds. Within the charged residue model (CRM) [13], the species participating in an equilibrium would have more time until the final ionization step because the analytes remain within the droplets until all solvent has evaporated. Today, most publications state [14] that both ion formation pathways coexist with a preference for one or the other depending on the properties of the analyte, such as its molecular weight. With increasing analyte mass, the CRM is expected to predominate, whereas for smaller molecules the IEM better explains experimental results [15].

The measurements presented here focus on larger droplets, with diameters in the micrometer range, down to about $1 \mu \mathrm{m}$. The data therefore relate to parent droplets and possible shifts of chemical equilibria as they shrink in size.

The effect of electrospray ionization on chemical equilibria was previously investigated by Wang and Agnes [16, 17]. By using ethylenediaminetetraacetatemetal complexation, it was shown that equilibria can be shifted during transfer to the gas phase as detected with the mass spectrometer. One of the first publications in which processes directly in the electrospray plume itself have been studied was published by Cook and coworkers [18]. By using laser-induced fluorescence the plume density was measured. Later, $\mathrm{pH}$ changes were investigated [19] by using fluorescent $\mathrm{pH}$ indicators. Protein denaturation in the electrospray aerosol was studied by monitoring fluorescence from a specific tryptophan residue in cytochrome C [20]. A study that addresses the possibility of equilibrium shifts within the electrospray was published by Scott and coworkers [21]. By measuring the fluorescence quenching of a fluorphore/ quencher system at two different positions within the spray, it was shown that the quenching rate is different at these positions and that it differs from quenching rates in solution. However, in these studies no information about the electrospray itself, such as droplet size, was available.

In this study, we combined spatially resolved fluorescence measurements with phase Doppler anemometry, which allows the measurement of droplet sizes, numbers, and velocities [22, 23]. The ${ }^{\circ}$ evolution ${ }^{\circ}$ of $^{\circ}$ an ${ }^{\circ}$ equilibrium ${ }^{\circ}$ in the spray plume with decreasing droplet size was probed. As a first model system we used rhodamine B-sulfonylchloride (RBS), which forms dimers at higher concentra- tions. To probe the concentration increase directly within the shrinking droplets, without any complexation kinetics involved, we used the fluorescent dyes DCM and oxazine 1. Fluorescence resonance energy transfer (FRET) between these gives a direct measure of the concentration increase within the droplets.

\section{Experimental}

Rhodamine B-sulfonylchloride was obtained from Acros Organics (Geel, Belgium). To prevent hydrolysis it was stored in dry form at $-20^{\circ} \mathrm{C}$. DCM [4dicyanomethylene-2-methyl-6-( $p$-dimethylaminostyril)$4 \mathrm{H}$-pyren] and oxazine 1 (3-diethylamino-7-diethyliminophenoxazonium derchlorate) were obtained from Lambda Physik (Göttingen, Germany). According to their spectral characteristics the dye pair should allow the observation of fluorescence resonance energy transfer as a function of their mutual distance (DCM $\lambda$ ex/ $\lambda \mathrm{em}=472 / 644 \mathrm{~nm}$; oxazine $1 \lambda \mathrm{ex} / \lambda \mathrm{em}=646 / 670 \mathrm{~nm})$ [24]..$^{\circ}$ Also, ${ }^{\circ}$ as ${ }^{\circ} a^{\circ}$ result ${ }^{\circ}$ of $^{\circ}$ the ${ }^{\circ}$ large ${ }^{\circ}$ Stokes ${ }^{\circ}$ shift $^{\circ}$ of ${ }^{\circ} \mathrm{DCM}$, the acceptor itself shows practically no absorption at the excitation wavelength. As solvent, HPLC-grade acetonitrile from Labscan Ltd. (Dublin, Ireland) was used. For fluorescence measurements in solution, an Aminco Bowman Series II Spectrofluorometer (Thermo Electron, Waltham, MA, USA) was used. Glass capillaries with $5 \mu \mathrm{l}$ sample volume were used as cuvettes.

The setup for ESI in situ measurements consisted of the electrospray and the fluorescence/PDA-measurement system $^{\circ}\left(\right.$ Figure $\left.^{\circ} 1\right){ }^{\circ}{ }^{\circ}$ For $^{\circ}$ the ${ }^{\circ}$ electrospray, ${ }^{\circ} a^{\circ}$ syringe ${ }^{\circ}$ pump (Harvard Apparatus, Holliston, MA, USA) was connected to the spray capillary with PEEK tubing. The stainless steel capillary had an i.d. of $0.40 \mathrm{~mm}$ and an o.d. of $0.68 \mathrm{~mm}$. The distance between the spray orifice and the counter electrode was $6 \mathrm{~cm}$. As counter electrode, a flat metal plate with a diameter of $30 \mathrm{~cm}$, which could be heated up to $280^{\circ} \mathrm{C}$, was used. Electrospray measurements were performed in positive mode by applying a voltage with a maximum $15 \mathrm{kV}$ adjustable DC power supply (Fug $\mathrm{GmbH}$, Rosenheim, Germany) to the capillary. The PDA (TSI Inc., St. Paul, MN, USA) was used to measure spray droplet sizes and velocities during ESI. It is operated with a water-cooled $5 \mathrm{~W}$ argon-ion laser (Innova 70, LA-70-5), generating a green and blue laser beam with a wavelength of $514.4^{\circ}$ and ${ }^{\circ} 488^{\circ} \mathrm{nm},{ }^{\circ}$ respectively ${ }^{\circ}[25]^{\circ} .^{\circ} \mathrm{The}^{\circ} \mathrm{PDA}^{\circ}$ and fluorescence detection optics are positioned by a threedimensional translation stage system (isel, 9450-XYZ500) controlled by a stepping motor (isel, C 142-4.1). For the detection of fluorescence within the electrospray plume a CCD spectrometer (Holospec, Kaiser Optical Systems Inc., Ann Arbor, MI, USA) with a wavelength range of 400 to $800 \mathrm{~nm}$ was used. For fluorescence detection a collecting lens was used that also served as fiber coupler for the connection to the CCD spectrometer. With this arrangement a detection volume of about $1 \mathrm{~mm}^{3}$ was achieved. The detection volumes for PDA and for fluorescence detection were adjusted to overlap with each other. 


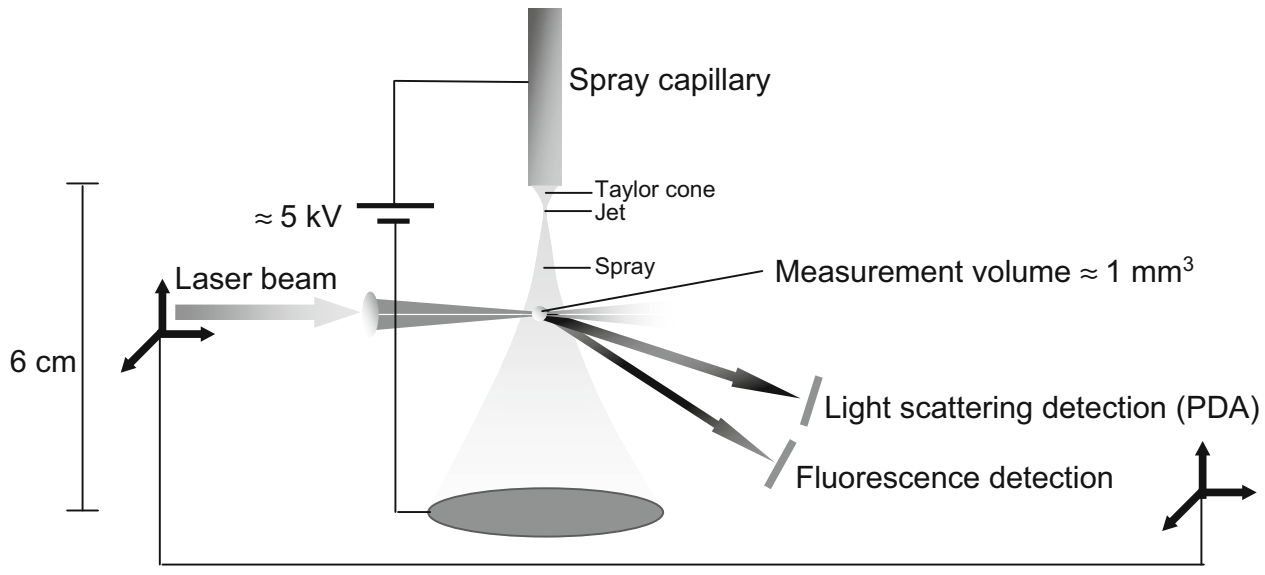

Figure 1. Setup with laser-induced fluorescence and phase Doppler anemometry. The argon ion laser is used for fluorescence excitation and as a light source for the phase Doppler anemometer. For the phase Doppler anemometer the laser is split into two beams, which are then crossed within the measurement volume. To use the same detection volume for the fluorescence detection, the detection optics for the fluorescence detection are focused into the same volume. Excitation and detection optics are mounted on the same $\mathrm{XYZ}$ positioning system. Droplets start to emerge at the beginning of the spray region.

\section{Results and Discussion}

The electrospray was operated in the stable conejet mode that shows a constant and narrow droplet size distribution, allowing acquisition times in a range from seconds to minutes, without averaging over different droplet $^{\circ}$ size $^{\circ}$ populations ${ }^{\circ}[26]^{\circ}$ The $^{\circ}$ prerequisite ${ }^{\circ}$ for ${ }^{\circ}$ a stable conejet mode is carefully adjusted parameters: The flow rate of liquid pumped through the capillary must exactly match the volume used by the electrospray. If the flow rate is higher or lower than required, fluctuations are introduced. Also, the solution used must have a certain conductivity and surface tension. Too high a conductivity and surface tension (such as water ${ }^{\circ}$ prevent $^{\circ}$ the $e^{\circ}$ formation ${ }^{\circ}$ of $^{\circ} \mathrm{a}^{\circ}$ stable $^{\circ}$ conejet $^{\circ}[27]$. The conejet mode could be obtained with 2-butanol and acetonitrile. With solvents such as methanol, ethanol, chloroform, and water, it was not possible to achieve a stable conejet with our setup. Here acetonitrile was used because it was a suitable solvent for all fluorescent dyes we intended to use. A typical droplet size distribution for acetonitrile and dissolved fluorescent dyes DCM and oxazine 1 at a concentration of $10 \mu \mathrm{M}$ each is shown ${ }^{\circ}{ }^{\circ}{ }^{\circ}{ }^{\circ}$ example $^{\circ}$ in ${ }^{\circ}$ Figure $^{\circ} 2$.

The size distribution measurements at different spray positions in positive and negative electrospray mode are shown. In positive mode, the data showed an electrospray with a starting droplet diameter of about $^{\circ} 7^{\circ} \mu \mathrm{m}^{\circ}$ in ${ }^{\circ}$ the $^{\circ}$ stable $^{\circ}$ conejet $^{\circ}$ mode $^{\circ}\left(\right.$ Figure $^{\circ} 2 \mathrm{a}$ ) shrinking ${ }^{\circ}$ down $^{\circ}$ to $^{\circ}$ about $^{\circ} 1^{\circ} \mu \mathrm{m} .{ }^{\circ}$ Figure $^{\circ} 2 \mathrm{~b}^{\circ}$ shows $^{\circ}$ an example in negative mode, where a stable conejet was not achieved, thus producing a broad droplet size distribution.

We carried out calculations to estimate the behavior of an equilibrium within a model droplet shrinking from 10 to $1 \mu \mathrm{m}$. The droplet volume as a function of time was determined from PDA experiments. For the on rate we assumed a typical diffusion-controlled reaction, which means a rate constant of about $10^{8} \mathrm{~s}^{-1} \mathrm{M}^{-1}$. For dissociation, we assumed different off rates between $10^{6}$ and $10^{-2} \mathrm{~s}^{-1}$, which cover the range from low- to high-affinity binding, corresponding to dissociation constants between $100 \mu \mathrm{M}$ and $0.1 \mathrm{nM}$, respectively. Strictly speaking, equilibrium constants $\mathrm{K}$ are dimensionless (according to the thermodynamic definition $\Delta G^{0}=-R T \ln K$, where $\left.K=\prod_{i} a_{i}^{k}\right)$. Here we adopt the phenomenological definition of equilibrium constants used in practice where activities $a_{i}$ are replaced by concentrations $c_{i}$. As a consequence, $\mathrm{K}$ will no longer be dimensionless; for example, the dissociation constant of a bimolecular reaction, $\mathrm{K}_{\mathrm{d}}=\left(\mathrm{c}_{\mathrm{A}} \mathrm{C}_{\mathrm{B}}\right) / \mathrm{c}_{\mathrm{C}}$, will have units of $\mathrm{mol} / \mathrm{L}$. Equation set 1 was used to calculate the equilibrium shift introduced by the shrinking droplet process. Because the volume is time dependent, we calculated the number of molecules per droplet rather than the concentrations for all species participating in the reaction $\mathrm{A}+\mathrm{B} \leftrightarrows \mathrm{C}$. Note that the model used here is simplified because it does not take into account droplet shrinking caused by the repeated Coulomb explosions. Starting concentrations for A and B were $100 \mu \mathrm{M}$, that is, $3.15 \times 10^{7}$ molecules per droplet. The results $^{\circ}$ are $^{\circ}$ shown $^{\circ}{ }^{\circ}{ }^{\circ}$ Figure $^{\circ} 3$.

$$
\begin{aligned}
& \frac{d n_{A}}{d t}=-\mathrm{k}_{1} \frac{n_{A} \cdot n_{B}}{\mathrm{~V}}+\mathrm{k}_{-1} \cdot \mathrm{n}_{\mathrm{C}}+\frac{\mathrm{n}_{\mathrm{A}}}{\mathrm{V}} \cdot \frac{d V}{d t} \\
& \frac{d n_{B}}{d t}=-\mathrm{k}_{1} \frac{n_{A} \cdot n_{B}}{\mathrm{~V}}+\mathrm{k}_{-1} \cdot \mathrm{n}_{\mathrm{C}}+\frac{\mathrm{n}_{\mathrm{B}}}{\mathrm{V}} \cdot \frac{d V}{d t} \\
& \frac{d n_{C}}{d t}=\mathrm{k}_{1} \frac{n_{A} \cdot n_{B}}{\mathrm{~V}}-\mathrm{k}_{-1} \cdot \mathrm{n}_{\mathrm{C}}+\frac{\mathrm{n}_{\mathrm{C}}}{\mathrm{V}} \cdot \frac{d V}{d t}
\end{aligned}
$$


(a)
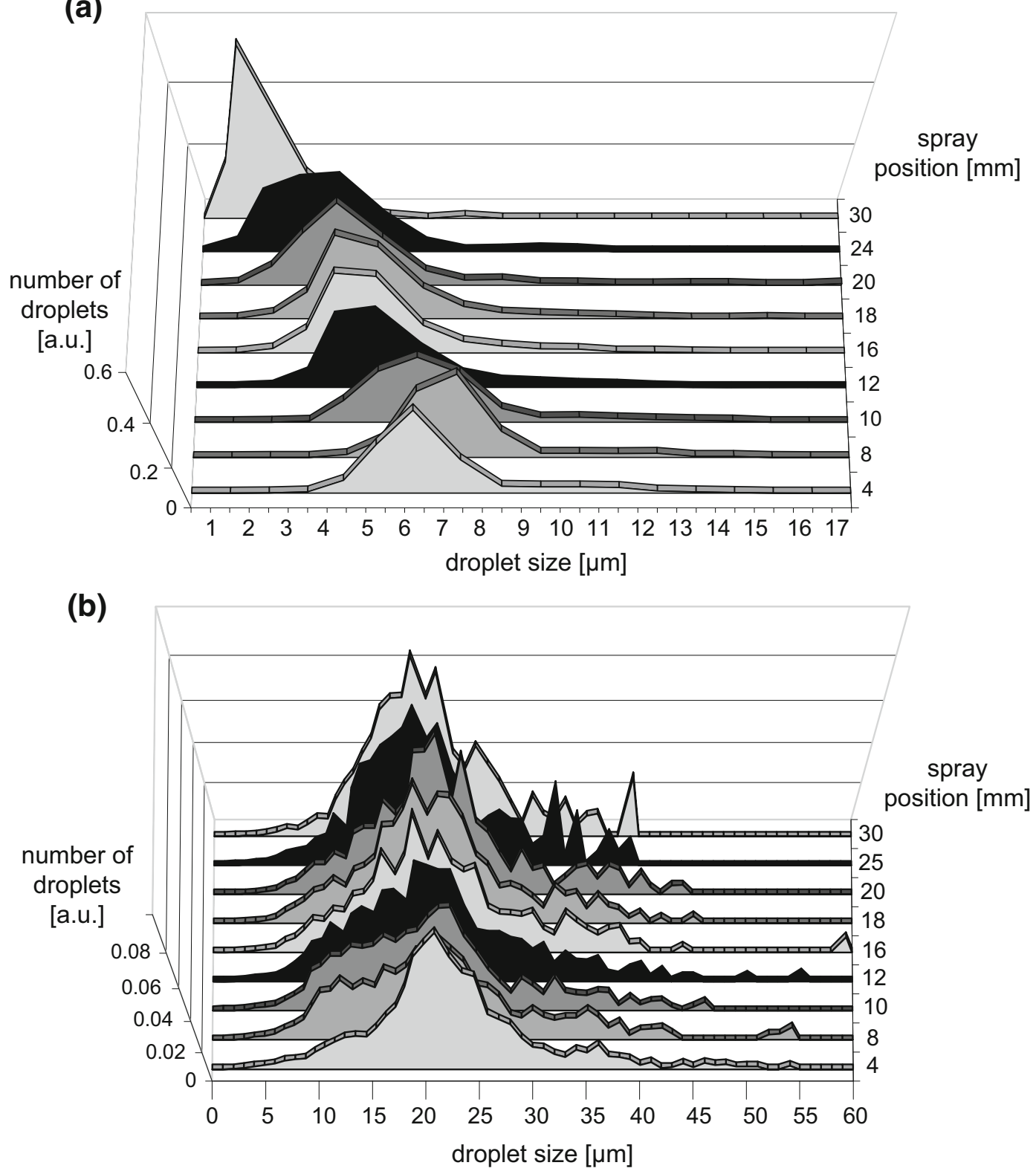

Figure 2. Droplet size distribution at different spray positions: comparison of positive (a) and negative (b) spraying mode for an acetonitrile solution containing $10 \mu \mathrm{M} \mathrm{DCM}$ and oxazine 1 . A stable conejet could be achieved only for the positive mode (see text), which is reflected in the narrow droplet size distribution (a). The spraying mode, which was the best result achieved in negative mode, is characterized by a broad droplet size distribution (b).

For the lower-affinity systems, that is, a $\mathrm{K}_{\mathrm{d}}$ of $10^{-4} \mathrm{M}$, the shrinking droplet volume has a significant effect, whereas for higher-affinity systems only limited influence is found. The latter is clear because for high-affinity systems almost all species are already complexed, so that an additional concentration increase has only a negligible effect. However, for a typical MS-based titration experiment, concentrations for which a considerable amount of unbound analyte exists would be used. For higher binding affinity systems, this is achieved simply by lowering the starting concentrations.
To experimentally investigate an equilibrium in a shrinking droplet a model system was chosen. From dye laser'researchit is known [28-30] that some organic fluorescent dyes tend to form dimers (and larger aggregates) at higher concentrations. These laser dyes typically have high quantum yields, are highly photostable, and ${ }^{\circ}$ are $^{\circ}$ soluble ${ }^{\circ}{ }^{\circ}$ organic $^{\circ}$ solvents ${ }^{\circ}[31,32]$. ${ }^{\circ}$ Rhodamine B-sulfonylchloride (RBS) was found to have the highest tendency to form dimers at higher concentrations (results not shown) and is soluble in acetonitrile. Also, its optical properties such as sufficient absorption at the 


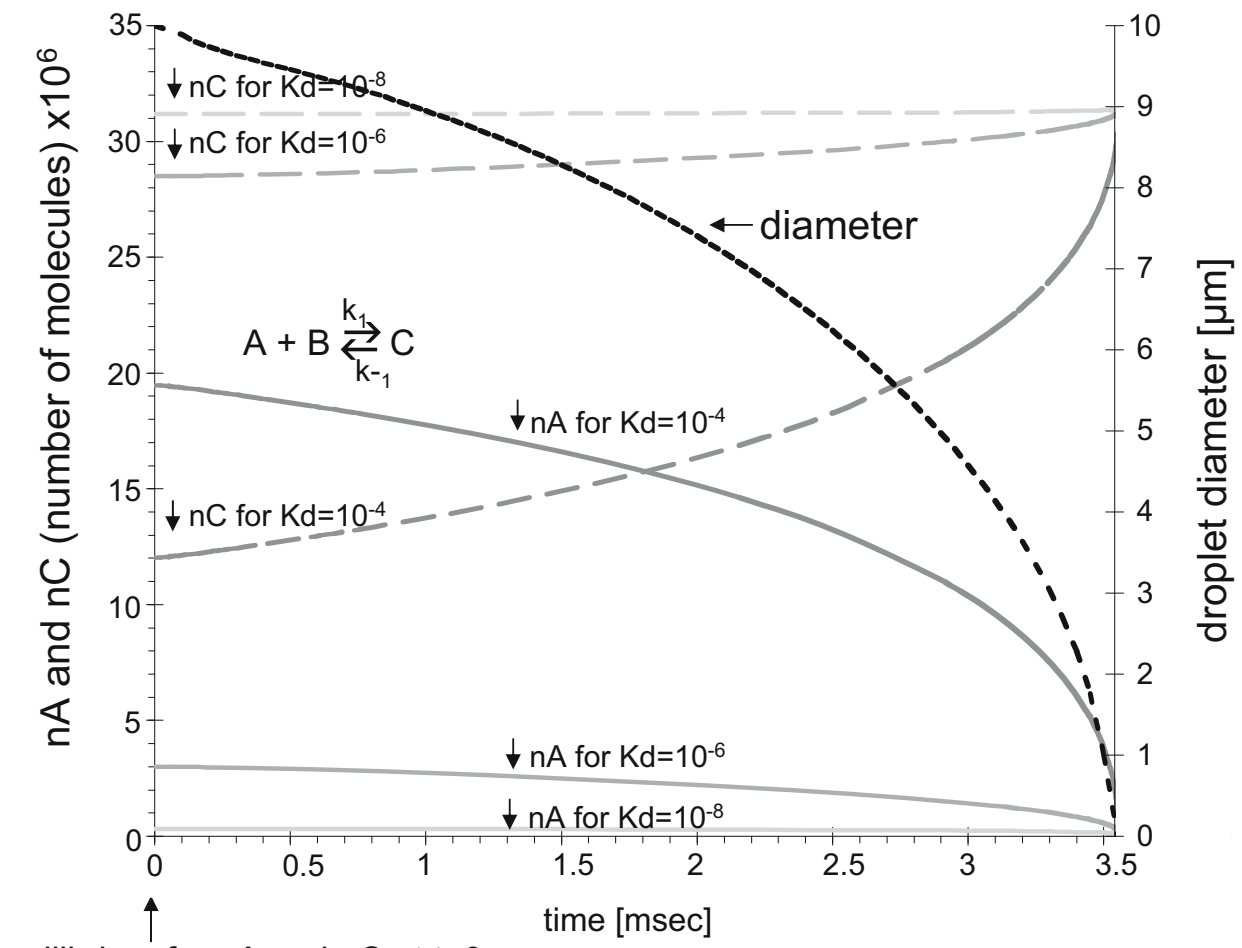

equilibrium for $\mathrm{nA}$ and $\mathrm{nC}$ at $\mathrm{t}=0$

Figure 3. Calculation of changes in the equilibrium for the reaction $\mathrm{A}+\mathrm{B} \leftrightarrow \mathrm{C}$ in a droplet shrinking from 10 to $1 \mu \mathrm{m}$. Initial concentrations of $\mathrm{A}$ and $\mathrm{B}$ are $100 \mu \mathrm{M}$, corresponding to $3.15 \times 10^{7}$ molecules each within a droplet of $10 \mu \mathrm{m}$ diameter. For the reaction an on-rate constant $\mathrm{k}_{1}$ of $10^{8} \mathrm{M}^{-1} \mathrm{~s}^{-1}$ was assumed, which is a typical value for a diffusion-controlled association, the off-rate constant $\mathrm{k}_{-1}$ varies according to the chosen dissociation constants $\mathrm{K}_{\mathrm{d}}$. For the plot the number of molecules was used instead of concentration, to avoid confusion because of the decreasing volume. The plot was created by numerical integration of equation set 1 .

laser wavelength available (514 nm) and high quantum yield are advantageous. Dimerization leads to a red shift of the fluorescence emission, and thus it is possible to ${ }^{\circ}$ follow ${ }^{\circ}$ the ${ }^{\circ}$ dimer $^{\circ}$ formation ${ }^{\circ}$ as $^{\circ} \mathrm{a}^{\circ}$ function ${ }^{\circ}$ of $^{\circ}$ concentration $^{\circ}$ in $^{\circ}$ solution $^{\circ}$ (Figure $\left.{ }^{\circ} 4\right)$.

The ${ }^{\circ}$ inset ${ }^{\circ}$ of ${ }^{\circ}$ Figure $^{\circ} 4^{\circ}$ shows ${ }^{\circ}$ the $e^{\circ}$ maximum ${ }^{\circ}$ emission wavelength of RBS solutions in acetonitrile at different concentrations up to $1 \mathrm{mM}$, which is close to the maximum solubility for this type of molecule in organic solvents [33]. The maximum ${ }^{\circ}$ mission ${ }^{\circ}$ wavelength shifts from $570 \mathrm{~nm}$ at $100 \mu \mathrm{M}$ to almost $598 \mathrm{~nm}$ at $1 \mathrm{mM}$ and above. Fluorescence measurements at such high concentrations ${ }^{\circ}$ in $^{\circ}$ solution $^{\circ}$ required ${ }^{\circ}$ the ${ }^{\circ}$ use $^{\circ}$ of $^{\circ} a^{\circ}$ special low-volume capillary cuvette to avoid inner filter effects that would otherwise lead to reabsorption of fluorescence ${ }^{\circ}$ photons ${ }^{\circ}[34]^{\circ}$. Plotting ${ }^{\circ}$ the ${ }^{\circ}$ maximum ${ }^{\circ}$ emission wavelength versus the concentration in a logarithmic plot yields a sigmoidic curve. Assuming that only monomer is present at low concentrations, whereas at high concentrations only dimers exist, a binding constant can be calculated $\left(\mathrm{K}_{\mathrm{d}}=90 \mu \mathrm{M}\right)$. This value can be compared with the $100 \mu \mathrm{M} \mathrm{K} \mathrm{C}_{\mathrm{d}}$ curve $^{\circ}$ in $^{\circ}$ Figure $^{\circ} 3 .^{\circ} \mathrm{To}$ estimate the concentration increase within the electrospray we considered a hypothetical spherical droplet shrinking from 10 to $1 \mu \mathrm{m}$ diameter, which is in the measurable range of the PDA. In this case, by a simple calculation, the concentration would increase by a factor of 1000. Therefore, for a starting concentration of $10 \mu \mathrm{M}$, which is a typical concentration used in ESI-MS experiments, already after a few milliseconds, millimolar concentrations would be reached. Whether these high concentrations lead to precipitation processes was not investigated, although precipitation of analyte was experimentally found in slowly evaporating droplets trapped ${ }^{\circ}{ }^{\circ}{ }^{\circ}{ }^{\circ}$ electrodynamic ${ }^{\circ}$ balance ${ }^{\circ}[35]$.

Based $^{\circ}$ on $^{\circ}$ Figure $^{\circ} 4,{ }^{\circ}$ the ${ }^{\circ}$ concentration $^{\circ}$ in ${ }^{\circ} a^{\circ}$ shrinking droplet that would lead to a significant red shift should already be reached for a droplet that shrunk to $50 \%$ of its original diameter. For example, with a $100 \mu \mathrm{M}$ starting concentration an emission wavelength of about $580 \mathrm{~nm}$ is expected; if a droplet with this concentration shrinks to half its diameter the resulting concentration of $0.8 \mathrm{mM}$ should result in an emission wavelength of $>590 \mathrm{~nm}$. However, this consideration neglects any kinetics for dimer association or spray processes other than solvent evaporation. For example, a slow association kinetic would reduce the effect of the fast concentration increase within the electrosprayed droplets.

Figure $^{\circ} 4^{\circ}$ shows $^{\circ}$ the ${ }^{\circ}$ fluorescence ${ }^{\circ}$ measurement ${ }^{\circ}$ of $^{\circ} \mathrm{a}$ $100 \mu \mathrm{M}$ solution of RBS in the ESI plume. The first $5 \mathrm{~mm}$ 


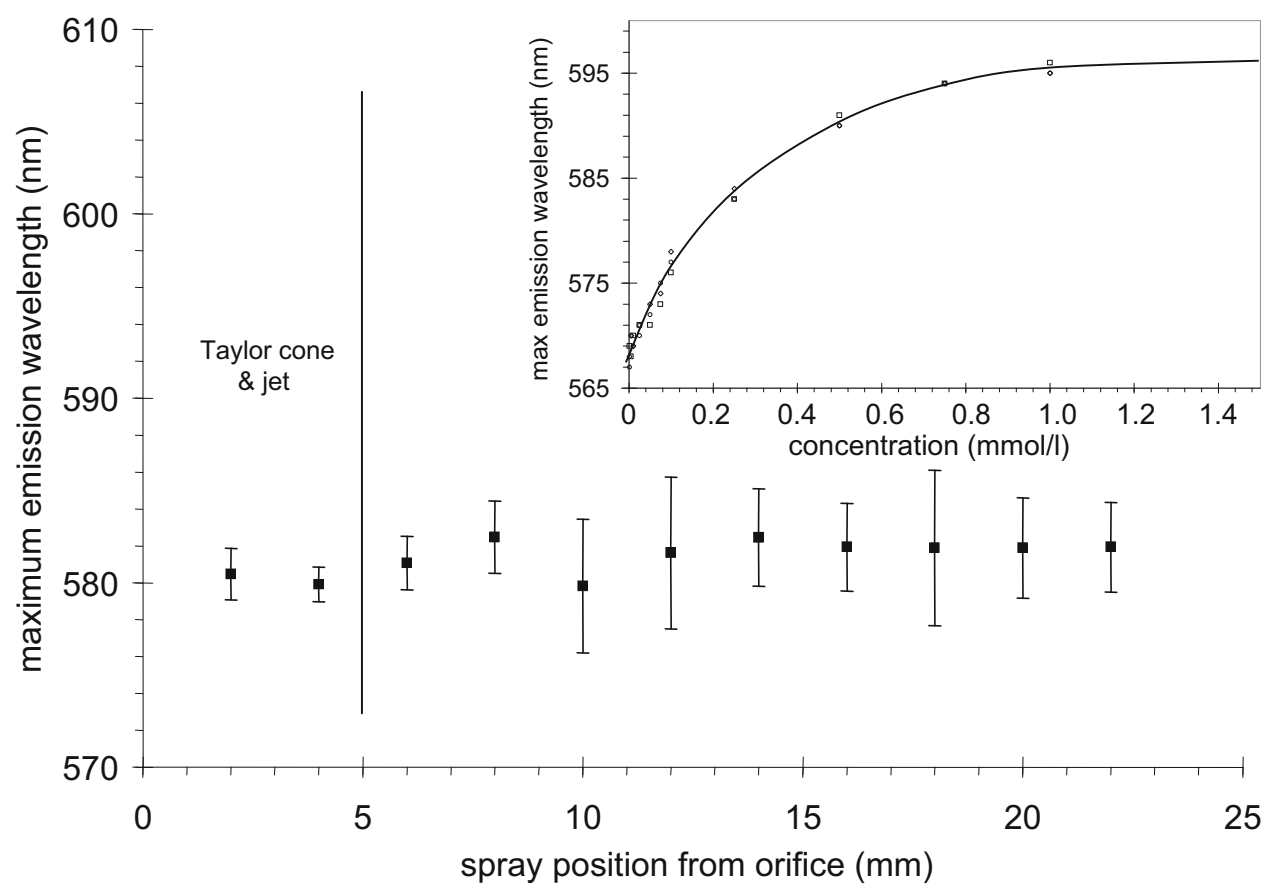

Figure 4. Fluorescence maximum for $100 \mu \mathrm{M}$ rhodamine B-sulfonylchloride at different positions within the electrospray plume. All measurements were done along the central axis of the (axially symmetric) spray. The first $4 \mathrm{~mm}$ of the electrospray consists of the Taylor cone and the jet; first droplets are measurable at about $5 \mathrm{~mm}$ away from the spray nozzle. At distances $>24 \mathrm{~mm}$ from the spray orifice, a fluorescence signal was no longer detected. Inset: Solution fluorescence emission of sulforhodamine B-sulfonylchloride in acetonitrile at different concentrations. These data were used for an estimation of the binding constant of the monomer/dimer equilibrium, $\mathrm{K}_{\mathrm{d}}=90 \mu \mathrm{M}$.

consist of the Taylor cone and the subsequent jet. First droplets emerged after about $6 \mathrm{~mm}$ from the spray capillary. The first detectable droplets, at a measurement position of $6 \mathrm{~mm}$ away from the spray orifice, had a size of about $11 \mu \mathrm{m}$ diameter. A fluorescence signal was detectable up to a distance of $22 \mathrm{~mm}$. At this distance the mean droplet diameter had decreased to $<2 \mu \mathrm{m}$. However, no shift in fluorescence emission wavelength was observed over this range, which implies no equilibrium shifts. This finding is unexpected, although it can be explained in several ways: (1) The short timescale of the electrospray: the velocity of the emitted droplets measured by PDA was about $5 \mathrm{~m} / \mathrm{s}$. From the beginning of the droplet creation until the last measurement point, a droplet spends only about $4 \mathrm{~ms}$. This time interval may be too short to allow the RBS monomer-dimer equilibrium to follow the concentration increase. (2) The production of small offspring droplets by Coulomb explosions: if a significant fraction of analyte is transported into the small offspring droplets during a fission event, the pressure on the equilibrium in the original droplet would be relieved. Offspring droplets are too small to be detected by the PDA system. (3) Cooling caused by solvent evaporation: to estimate this effect, we performed a calculation for an acetonitrile droplet shrinking from 10 to $1 \mu \mathrm{m}$ within the timescale observed in the PDA experiments. The model for evaporation originally introduced by Abramzon $^{\circ}$ and ${ }^{\circ}$ Sirignano $^{\circ}[36]^{\circ}$ is $^{\circ}$ described $^{\circ}$ in $^{\circ}$ detail $^{\circ}$ by ${ }^{\circ}$ Heine and ${ }^{\circ}$ Pratsinis ${ }^{\circ}[25] .{ }^{\circ}$ The ${ }^{\circ}$ temperature ${ }^{\circ}$ within ${ }^{\circ}$ the ${ }^{\circ}$ droplets was estimated to decrease by $>20 \mathrm{~K}$. This significant temperature drop could result in "freezing" of the equilibrium. To further investigate this possibility, we carried out a fluorescence experiment using a heated plate as counter electrode, to avoid freezing of the equilibrium. However, even at a plate temperature of $200{ }^{\circ} \mathrm{C}$, no emission shift was observable.

A key question therefore was whether it is possible at all to observe a dynamic concentration increase arising from volume reduction of droplets within the electrospray. We decided to use as indicators the fluorescent dyes DCM and oxazine 1. This pair allows measurement of an average distance by fluorescence energy transfer. The DCM serves as donor and oxazine 1 as acceptor. Both dyes are very suitable for these experiments because they are typically ${ }^{\circ}$ sed $^{\circ}$ as 9 laser ${ }^{\circ}$ dyes $[37]^{\circ}$ because ${ }^{\circ}$ of high photosta- $^{\circ}$ bility and high fluorescence quantum yield.

Figure $^{\circ} 5 \mathrm{a}^{\circ}$ shows ${ }^{\circ}$ the ${ }^{\circ}$ calculation ${ }^{\circ}$ of $^{\circ}$ average $e^{\circ}$ intermolecular distances with increasing concentration and an example for the efficiency of FRET, as a function of mutual distance. This calculation is only an estimation of the concentrations that are necessary to observe energy transfer. In solution, the distance between dye molecules is not constant as a result of diffusional motion. In electrospray droplets the situation is even more complex because analytes can be distributed inhomogeneously, as mentioned earlier in the introduction. 

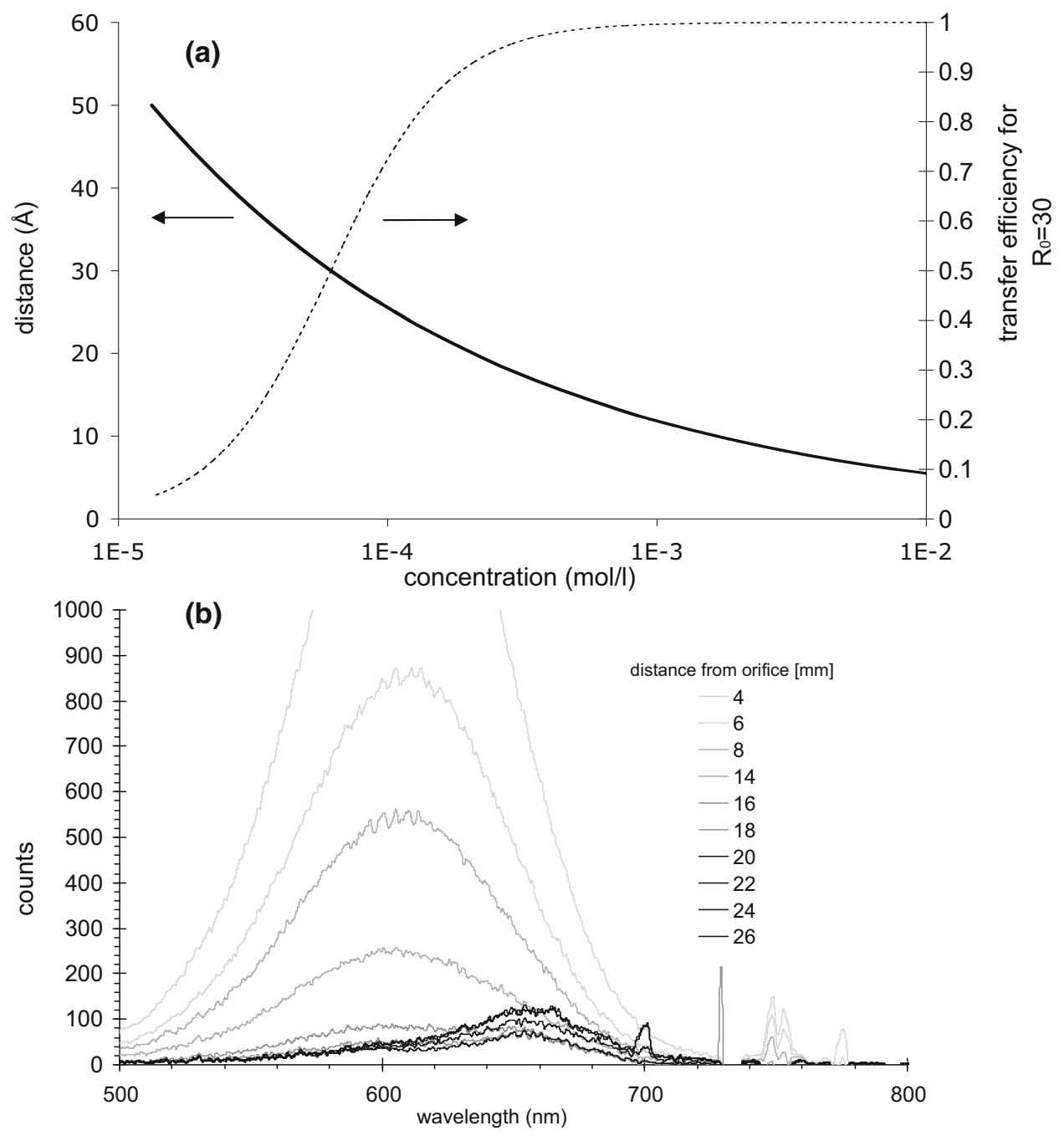

Figure 5. (a) Calculation of the maximum distance of two molecules at a given concentration (black line, left $y$-axis). Also the energy-transfer efficiency for a donor acceptor pair is plotted for which the distance where energy-transfer efficiency is $50 \%$ is $30 \AA$ (dotted line, right axis). It gives a rough estimate for the concentrations needed to observe fluorescence energy-transfer. (b) Measurement of a $10 \mu \mathrm{M}$ DCM/10 $\mu \mathrm{M}$ oxazine 1 solution at different positions within the spray. The appearance of a second fluorescence peak shows the occurrence of fluorescence energy transfer, about $18 \mathrm{~mm}$ from the orifice.

The FRET rate depends on the inverse 6th power of the distance, so the rate of energy transfer is expected to drastically increase with decreasing distance between molecules. Within our measurement volume, FRET will give an average value for the proximity of fluorescent dyes. A concentration of $10 \mu \mathrm{M}$ for donor and acceptor was used for the electrospray, resulting in a stable conejet and a sufficiently high fluorescence intensity. Figure ${ }^{\circ} 5 b^{\circ}$ shows $^{\circ}$ the ${ }^{\circ}$ fluorescence ${ }^{\circ}$ spectra ${ }^{\circ}$ recorded $^{\circ}$ at different positions in the spray. The overall fluorescence intensity decreases with increasing distance from the spray orifice. The relative intensities of the fluorescence emission maxima for both dyes change with decreasing droplet size. The latter finding clearly demonstrates that it is possible to follow the dynamic processes within the electrospray using FRET. To evaluate the efficiency of fluorescence energy transfer and thus the average dis- tance or "concentration" of molecules, the fluorescence intensity of donor relative to the intensity of the acceptor was calculated and then plotted against the measurement ${ }^{\circ}$ position $^{\circ}{ }^{\text {within }}{ }^{\circ}$ the ${ }^{\circ}$ spray $^{\circ}\left(\right.$ Figure $\left.^{\circ} 6 a\right)$.

The slope reduction beginning at $20 \mathrm{~mm}$ results from the decreasing signal intensity. The corresponding droplet sizes areshown in Figure $6 \mathrm{~b}$. The 9 argest ${ }^{\circ}$ droplet size was measured at the beginning of the spray, as expected, with an average droplet diameter of $7 \mu \mathrm{m}$. Within $25 \mathrm{~mm}$ along the central spray axis the average droplet diameter decreases to $<2 \mu \mathrm{m}$. Comparison of the results from the FRET experiments for solution and electrospray suggests that within the droplets, the analyte concentration increases by a factor of at least 100 . This $^{\circ}$ is ${ }^{\circ}$ in $^{\circ}$ good ${ }^{\circ}$ agreement ${ }^{\circ}$ with $^{\circ} \mathrm{Kebarle}^{\circ}$ and ${ }^{\circ} \mathrm{Ho}^{\circ}[38]$, who calculated a concentration increase by a factor of about 50, although for smaller droplets. However, it 

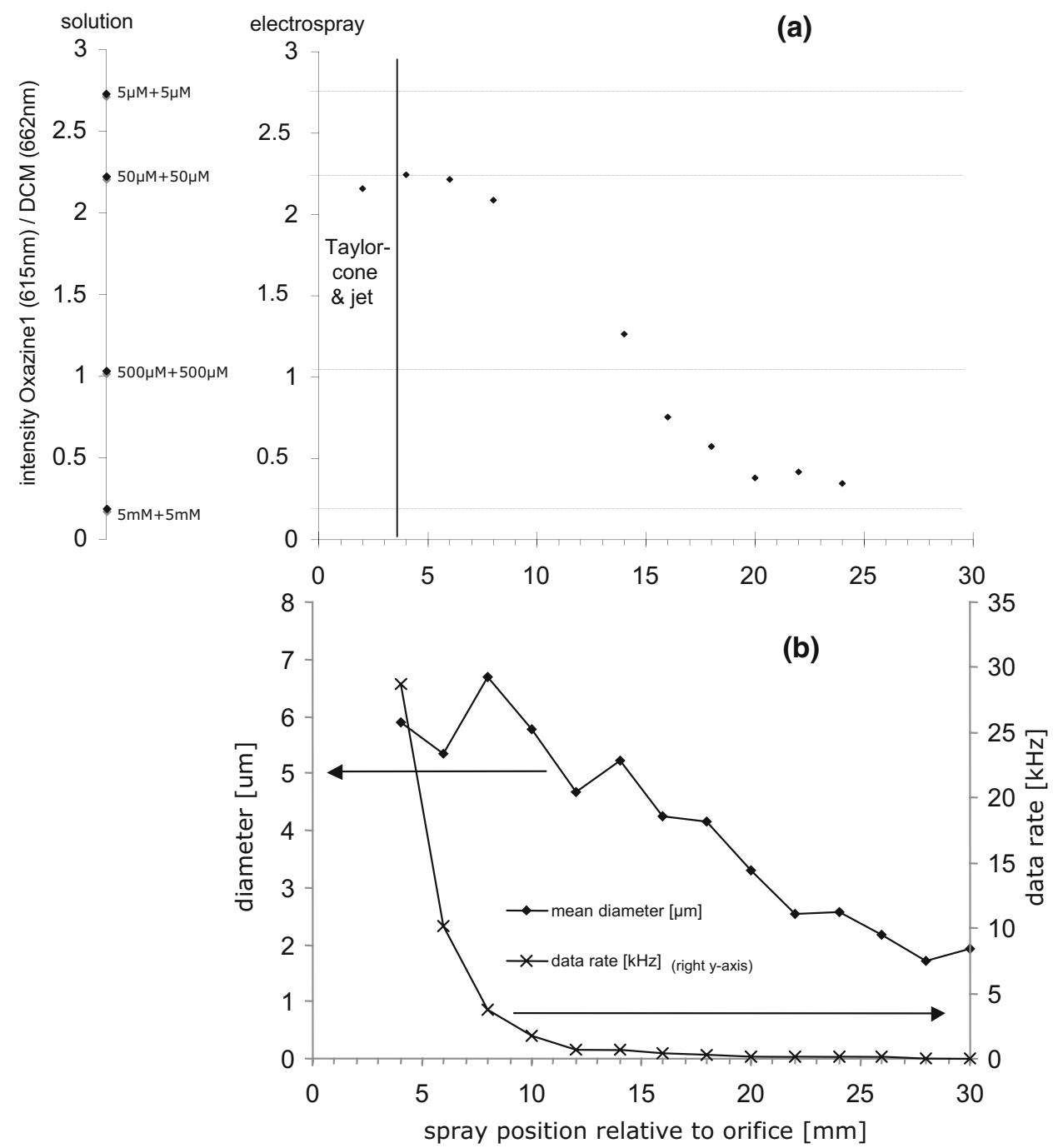

Figure 6. (a) Plot of the relative peak intensities for DCM- and oxazine-fluorescence, measured in solution and within the electrospray. With increasing distance from the spray orifice, the relative fluorescence emission from oxazine is increasing (thus the smaller ratio for DCM/oxazine). This is a measure for the increasing average proximity of both dyes. In (b) the corresponding droplet sizes are shown. At a distance of about $25 \mathrm{~mm}$ the fluorescence intensity is too low for further measurements.

should be noted here that the direct comparison between our solution and electrospray fluorescence data is only qualitative because of effects associated with fluorescence measurements at high concentrations in solution and because of spray phenomena that do not occur in solution, such as partitioning effects.

\section{Conclusions and Outlook}

To our knowledge this is the first study using fluorescence measurements in an electrospray with the additional information about droplet size and velocity, obtained by phase Doppler anemometry. We have investigated equilibrium processes directly within the first stages of the electrospray down to a droplet size of $1 \mu \mathrm{m}$. It was possible to follow the concentration increase within the shrinking droplets with a pair of fluorescent dyes (DCM and oxazine 1), using fluores- cence resonance energy transfer as an average distance indicator. For sulforhodamine B-sulfonylchloride, however, no influence of the electrospray on dimerization was observed, based on the absence of any shift in fluorescence emission. Although later stages of the electrospray with smaller droplets could not be probed, it is important information that at least in the primary droplets no influence arising from the shrinking droplet volume on a model equilibrium was found.

The experimental approach presented here could also be used to investigate other aspects of the electrospray such as partitioning processes. By using fluorescent dye systems with different combinations of charges in solution it should be possible to investigate how differently charged molecules distribute after Coulomb explosions within the electrospray; also the difference between positive and negative electrospray ionization could be investigated this way. Very interesting studies 
will be possible if the sensitivity of the fluorescence detection can be increased. It may be possible to follow the electrospray until the release of ionized analytes and, in this fashion, to obtain a full picture about the processes involved. Finally, the formation of a stable electrospray with a narrow and reproducible droplet size distribution should be optimized, such as by using piezo devices or a sheath gas flow, to facilitate droplet creation. This could make it possible to investigate aqueous sprays that would appreciably broaden the possibilities of the presented methodology.

\section{Acknowledgments}

The authors thank Robert J. Pfab, Gero Wortman, Eduard Miloglyadov, and Marina Introvigne for helpful discussions and technical assistance. Financial support for this work from the Swiss National Science Foundation (Grants 200020-103616 and $-107947 / 1)$ is gratefully acknowledged.

\section{References}

1. Ganem, B.; Li, Y. R.; Henion, J. D. Detection of Noncovalent ReceptorLigand Complexes by Mass Spectrometry. J. Am. Chem. Soc. 1991, 113, 6294-6296.

2. Ayed, A.; Krutchinsky, A. N.; Ens, W.; Standing, K. G.; Duckworth, H. W. Quantitative Evaluation of Protein-Protein and Ligand-Protein Equilibria of a Large Allosteric Enzyme by Electrospray Ionization Time-of-Flight Mass Spectrometry. Rapid Commun. Mass Spectrom. 1998, $12,339-344$.

3. Kraunsoe, J. A.; Aplin, R. T.; Green, B.; Lowe, G. An investigation of the binding of protein proteinase inhibitors to trypsin by electrospray Ionization Mass Spectrometry. FEBS Lett. 1996, 396, 108-112.

4. Daniel, J. M.; McCombie, G.; Wendt, S.; Zenobi, R. Mass Spectrometric Determination of Association Constants of Adenylate Kinase with Two Noncovalent Inhibitors. J. Am. Soc. Mass Spectrom. 2003, 14, 442-448.

5. Cole, R. B., Ed. Electrospray Ionization Mass Spectrometry: Fundamentals, Instrumentation, and Applications; Wiley: New York, 1997.

6. Enke, C. G. A Predictive Model for Matrix and Analyte Effects in Electrospray Ionization of Singly-Charged Ionic Analytes. Anal. Chem. 1997, 69, 4885-4893

7. Sjoberg, P. J.; Bokman, C. F.; Bylund, D.; Markides, K. E. A Method for Determination of Ion Distribution within Electrosprayed Droplets. Anal. Chem. 2001, 73, 23-28.

8. Sherman, C. L.; Brodbelt, J. S. An Equilibrium Partitioning Model for Predicting Response to Host-Guest Complexation in Electrospray Ionization Mass Spectrometry. Anal. Chem. 2003, 75, 1828-1836.

9. Tang, K.; Gomez, A. On the Structure of an Electrostatic Spray of Monodisperse Droplets. Phys. Fluids 1994, 6, 2317-2332.

10. Wilhelm, O.; Madler, L.; Pratsinis, S. E. Electrospray Evaporation and Deposition. J. Aerosol Sci. 2003, 34, 815-836.

11. Tang, K.; Smith, R. D. Physical/Chemical Separations in the Break-up of Highly Charged Droplets from Electrosprays. J. Am. Soc. Mass Spectrom. 2001, 12, 343-347.

12. Iribarne, J. V.; Thomson, B. A. On the Evaporation of Small Ions from Charged Droplets. J. Chem. Phys. 1976, 64, 2287-2294.
13. Dole, M.; Mack, L. L.; Hines, R. L.; Mobley, R. C.; Ferguson, L. D.; Alice, M. B. Molecular Beams of Macroions. J. Chem. Phys. 1968, 49, 2240-2249.

14. Kebarle, P.; Peschke, M. On the Mechanisms by Which the Charged Droplets Produced by Electrospray Lead to Gas Phase Ions. Anal. Chim. Acta 2000, 406, 11-35.

15. Kebarle, P. A Brief Overview of the Present Status of the Mechanisms Involved in Electrospray Mass Spectrometry. J. Mass Spectrom. 2000, 35, 804-817.

16. Wang, H. J.; Agnes, G. R. Evaluation of Electrospray Mass Spectrometry as a Technique for Quantitative Analysis of Kinetically Labile Solution Species. Anal. Chem. 1999, 71, 3785-3792.

17. Wang, H.; Agnes, G. R. Kinetically Labile Equilibrium Shifts Induced by the Electrospray Process. Anal. Chem. 1999, 71, 4166-4172.

18. Zhou, S.; Cook, K. D. Probing Solvent Fractionation in Electrospray Droplets with Laser-induced Fluorescence of a Solvatochromic Dye. Anal. Chem. 2000, 72, 963-969.

19. Zhou, S.; Prebyl, B. S.; Cook, K. D. Profiling $\mathrm{pH}$ Changes in the Electrospray Plume. Anal. Chem. 2002, 74, 4885-4888.

20. Rodriguez-Cruz, S. E.; Khoury, J. T.; Parks, J. H. Protein Fluorescence Measurements within Electrospray Droplets. J. Am. Soc. Mass Spectrom. 2001, 12, 716-725.

21. Ham, J. E.; Durham, B.; Scott, J. R. Design of Instrumentation for Probing Changes in Electrospray Droplets via the Stern-Volmer Relationship. Rev. Sci. Instrum. 2005, 76, 014101.

22. Olumee, Z.; Callahan, J. H.; Vertes, A. Droplet Dynamics Changes in Electrostatic Sprays of Methanol-Water Mixtures. J. Phys. Chem. A 1998 102, 9154-9160.

23. Smith, R. D.; Flagan, R. C.; Beauchamp, J. L. Droplet Evaporation and Discharge Dynamics in Electrospray Ionization. J. Phys. Chem. A 2002, 106, 9957-9967.

24. Stryer, L.; Haugland, R. P. Energy Transfer: A Spectroscopic Ruler. Proc. Natl. Acad. Sci. U.S.A. 1967, 58, 719-726.

25. Heine, M. C.; Pratsinis, S. E. Droplet and Particle Dynamics during Flame Spray Synthesis of Nanoparticles. Ind. Eng. Chem. Res. 2005, 44, 6222-6232.

26. Cloupeau, M.; Prunetfoch, B. Electrohydrodynamic Spraying Functioning Modes-A Critical Review. J. Aerosol Sci. 1994, 25, 1021-1036.

27. Lopez-Herrera, J. M.; Barrero, A.; Boucard, A.; Loscertales, I. G.; Marquez, M. An Experimental. Study of the Electrospraying of Water in Air at Atmospheric Pressure. J. Am. Soc. Mass Spectrom. 2004, 15, 253-259.

28. Holmes, W. C. The Influence of Variation in Concentration on the Absorption Spectra of Dye Solutions. Ind. Eng. Chem. 1924, 16, 35.

29. Levshin, L. V.; Gorskov, V. K. Opt. Spectrosc. 1961, 10, 401-405.

30. Selwyn, J. E.; Steinfeld, J. I. Aggregation Equilibria of Xanthene Dyes. J. Phys. Chem. 1972, 76, 762-774.

31. Levshin, L. V.; Lonskaya, I. S. Dependence of the Association of Rhodamines on the Structure of Their Molecules and the Nature of the Solvent. Opt. Spectrosc. 1961, 11, 148-150.

32. Levshin, L. V.; Slavnova, T. D.; Yuzhakov, V. I. Spectroscopic Manifestation of the Association of Rhodamine 6G in Alcoholic Solutions at Different Temparatures. Zh. Prikl. Spektrosk. 1976, 24, 985-990.

33. Lu, Y.; Penzkofer, A. Absorption Behaviour of Methanolic Rhodamine 6G Solutions at High Concentration. Chem. Phys. 1986, 107, 175-184.

34. Burghardt, T. P.; Lyke, J. E.; Ajtai, K. Fluorescence Emission and Anisotropy from Rhodamine Dimers. Biophys. Chem. 1996, 59, 119-131.

35. Bakhoum, S. F. W.; Agnes, G. R. Study of Chemistry in Droplets with Net Charge before and after Coulomb Explosion: Ion-Induced Nucleation in Solution and Implications for Ion Production in an Electrospray. Anal. Chem. 2005, 77, 3189-3197.

36. Abramzon, B.; Sirignano, W. A. Droplet Vaporization Model for Spray Combustion Calculations. Int. J. Heat. Mass Transfer 1989, 32, 1605-1618.

37. Drexhage, K. H. Dye Lasers; F. P. Schäfer, Ed.; Springer-Verlag: New York, 1977.

38. Kebarle, P.; Ho, Y. On the Mechanism of Electrospray Ionization; R. Cole, Ed.; Wiley-Interscience: New York, Chapter 1, 1997, pp 3-63. 